\title{
Global Analysis of a (1,2)-Type System of Non-Linear Difference Equations
}

\author{
Emine Yener $^{1}$, Mehmet Gümüş ${ }^{2 \star}$
}

\section{Abstract}

This paper deals with the study of global analysis of following $(1,2)$-type system of non-linear difference equations:

$$
u_{n+1}=\frac{\alpha v_{n-1}}{\beta+\gamma v_{n}^{p} v_{n-2}^{q}}, \quad v_{n+1}=\frac{\alpha_{1} u_{n-1}}{\beta_{1}+\gamma_{1} u_{n}^{p} u_{n-2}^{q}}, \quad n=0,1, \ldots
$$

where the parameters $\alpha, \beta, \gamma, \alpha_{1}, \beta_{1}, \gamma_{1, p}, q$ and the initial conditions $u_{i}, v_{i}, i=-2,-1,0$ are non negative real numbers.

Keywords: (1,2)-type system, Difference equations, Equilibrium, Global stability, Rate of convergence. 2010 AMS: 39A10, 40A05

${ }^{1}$ Zonguldak Bülent Ecevit University, Faculty of Science and Arts, Department of Mathematics, 67100, Zonguldak, Turkey ${ }^{2}$ Zonguldak Bülent Ecevit University, Faculty of Science and Arts, Department of Mathematics, 67100, Zonguldak, Turkey *Corresponding author: m.gumus@beun.edu.tr Received: 7 December 2020, Accepted: 28 December 2020, Available online:29 March 2021

\section{Introduction}

Difference equations (also called recursive sequences) appear in a lot of fields of pure and applied mathematics, both as discrete analogs of continuous behavior (analysis, numerical approximations) and as independent models for discrete behavior (population dynamics, economics, biology, ecology, etc.), [1]. In recent years, many models, especially in mathematical biology, are based on non-linear ones, [11]. Difference equation theory, especially nonlinear ones, is very fertile subject for scientists and is one of the important subjests of applied mathematics. So, many researchers have dealth with the qualitative behavior of nonlinear higher order rational difference equations and systems, see [1]-[33].

In [9], El-Owaidy et al. studied the global analysis of the following difference equation

$$
x_{n+1}=\frac{\alpha x_{n-1}}{\beta+\gamma x_{n-2}^{p}}, \quad n=0,1, \ldots
$$

with non-negative parameters and non-negative initial values.

In [5], Ahmed investigated the global asymptotic behavior and the perodic character for the rational difference equation

$$
x_{n+1}=\frac{b x_{n-1}}{A+B x_{n}^{p} x_{n-2}^{p}}, \quad n=0,1, \ldots
$$

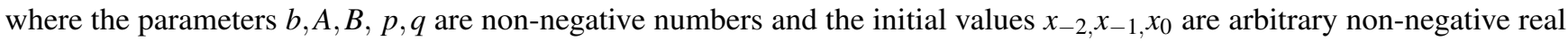
numbers. 
In [15], Gümüş and Soykan investigated the local asymptotic stability of equilibria, the periodic nature of solutions, the existence of unbounded solutions and the global behavior of solutions of the difference equation

$$
x_{n+1}=\frac{\alpha x_{n-(k+1)}}{\beta+\gamma x_{n-k}^{p} x_{n-(k+2)}^{q}}, \quad n=0,1, \ldots
$$

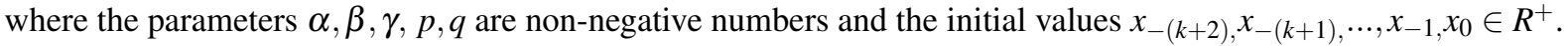

In [13], Gümüş and Soykan studied the dynamical behavior of positive solutions for a system of rational difference equation following form

$$
u_{n+1}=\frac{\alpha u_{n-1}}{\beta+\gamma v_{n-2}^{p}}, \quad v_{n+1}=\frac{\alpha_{1} v_{n-1}}{\beta_{1}+\gamma_{1} u_{n-2}^{p}}, \quad n=0,1, \ldots
$$

where the parameters $\alpha, \beta, \gamma, \alpha_{1}, \beta_{1}, \gamma_{1}, p$ and the initial values $u_{-i}, v_{-i}$ for $i=0,1,2$ are positive real numbers.

In [14], Gümüs and Öcalan studied the dynamical behavior of positive solutions for a system of rational difference equations following form

$$
u_{n+1}=\frac{\alpha u_{n-1}}{\beta+\gamma v_{n}^{p} v_{n-2}^{q}}, \quad v_{n+1}=\frac{\alpha_{1} v_{n-1}}{\beta_{1}+\gamma_{1} u_{n}^{p_{1}} u_{n-2}^{q_{1}}}, \quad n=0,1, \ldots
$$

where the parameters $\alpha, \beta, \gamma, \alpha_{1}, \beta_{1}, \gamma_{1}, p, q, p_{1}, q_{1}$ are positive real numbers and the initial values $u_{-i}, v_{-i}$ are non-negative real numbers for $i=0,1,2$.

In [28], Khan et al. investigated the asymptotic behavior of following anti-competitive system of rational difference equations

$$
\left.\begin{array}{c}
x_{n+1}=\frac{\alpha y_{n}}{\beta+\gamma x_{n}^{r}}, \\
y_{n+1}=\frac{\alpha_{1} x_{n}}{\beta_{1}+\gamma_{1} y_{n}^{t}},
\end{array}\right\}, n=0,1, \ldots
$$

where the parameters $\alpha, \beta, \gamma, \alpha_{1}, \beta_{1}, \gamma_{1}, r \in(0, \infty)$ and $x_{0}, y_{0} \in(0, \infty)$.

In [29], Qureshi and Din investigated the qualitative asymptotic behavior of positive solution for an anti-competitive system of third-order rational difference equations

$$
\left.\begin{array}{c}
x_{n+1}=\frac{y_{n-2}}{\beta+\gamma x_{n} x_{n-1} x_{n-2}}, \\
y_{n+1}=\frac{\alpha_{1} x_{n-2}}{\beta_{1}+\gamma_{1} y_{n} y_{n-1} y_{n-2}},
\end{array}\right\}, n=0,1, \ldots
$$

where the parameters $\alpha, \beta, \gamma, \alpha_{1}, \beta_{1}, \gamma_{1}$ and $x_{0}, x_{-1}, x_{-2}, y_{0}, y_{-1}, y_{-2}$ are positive real numbers.

In [27], Qureshi and Khan studied the global dynamics of following $(1,2)$-type systems of difference equations

$$
\begin{aligned}
& x_{n+1}=\frac{\eta y_{n-1}}{1+\mu x_{n-2}^{p}}, \quad y_{n+1}=\frac{\mu x_{n-1}}{1+\eta y_{n-2}^{p}}, \quad n=0,1, \ldots \\
& x_{n+1}=\frac{\eta y_{n-1}}{1+\mu y_{n-2}^{p}}, \quad y_{n+1}=\frac{\mu x_{n-1}}{1+\eta x_{n-2}^{p}}, \quad n=0,1, \ldots
\end{aligned}
$$

where $\eta, \mu, p$ and initial conditions $x_{l}, y_{l}, l=-2,-1,0$ are non negative real numbers.

In the present paper, we will investigate of some properties, such as the local asymptotic stability, the global asymptotic stability, the existence of periodic solutions, the rate of converges etc., for $(1,2)$-type system of difference equations in the title, which has been investigated different versions of it in the known literarture. We first note down critical error for the results of the article [27]. Namely, to put it briefly, they can not obtain the equations they claim with their transformations. Using the transformations, they could get equations in this form;

$$
\left.\begin{array}{c}
x_{n+1}=\frac{\eta y_{n-1}}{1+\mu y_{n-2}^{p}}, \\
y_{n+1}=\frac{\eta_{1} x_{n-1}}{1+\mu_{1} x_{n-2}^{p}},
\end{array}\right\}, n=0,1, \ldots
$$


with

$$
\eta=\frac{\alpha}{\beta}, \quad \eta_{1}=\frac{\alpha_{1}}{\beta_{1}}
$$

and

$$
\mu_{1}=\frac{\beta}{\gamma}, \mu=\frac{\beta_{1}}{\gamma_{1}}
$$

The same applies to the other equation. Let us also note that the theoretical results they obtained in their article are correct. However, an error was made only at the beginning.

The aim of this paper is to investigate the equilibrium points, the local asymptotic stability of these points, the global behavior of positive solutions, the existence of the prime two-periodic solutions and the rate of convergence of positive solutions of the following system

$$
\left.\begin{array}{c}
u_{n+1}=\frac{\alpha v_{n-1}}{\beta+\gamma v_{n}^{p} v_{n-2}^{q}}, \\
v_{n+1}=\frac{\alpha_{1} u_{n-1}}{\beta_{1}+\gamma_{1} u_{n}^{p} u_{n-2}^{q}},
\end{array}\right\}, n=0,1, \ldots
$$

where the parameters $\alpha, \beta, \gamma, \alpha_{1}, \beta_{1}, \gamma_{1}, p, q$ are positive and initial condition $u_{-2}, u_{-1}, u_{0}, v_{-2}, v_{-1}, v_{0} \in(0, \infty)$. Our results extend and complement some results in the literature.

If the initials conditions $u_{i}=v_{i}$ in the system (1.7) for $i \in\{-2,-1,0\}$ and $\alpha=\alpha_{1}, \beta=\beta_{1}, \gamma=\gamma_{1}$ then one obtain that $u_{n}=v_{n}$ for all $n \geqslant-2$, hence, the system (1.7) reduces to the difference equation

$$
v_{n+1}=\frac{\alpha v_{n-1}}{\beta+\gamma v_{n}^{p} v_{n-2}^{q}}, n=0,1, \ldots
$$

which was studied by [4]. Therefore, to avoid degenerate situations, here we discuss the case $u_{i} \neq v_{i}$ for $i \in\{-2,-1,0\}$ and we investigate the system (1.7) basing on this condition.

It is clear that the system (1.7) can be reduced to the following system of difference equations

$$
\left.\begin{array}{c}
x_{n+1}=\frac{r y_{n-1}}{1+s_{1} y_{n}^{p} y_{n-2}^{q}}, \\
y_{n+1}=\frac{r_{1} x_{n-1}}{1+s x_{n}^{p} x_{n-2}^{q}},
\end{array}\right\}, n=0,1, \ldots
$$

by the change of variables

$$
u_{n}=\left(\frac{\beta \beta_{1}}{\gamma \gamma_{1}}\right)^{1 / p+q} x_{n}
$$

and

$$
v_{n}=\left(\frac{\beta \beta_{1}}{\gamma \gamma_{1}}\right)^{1 / p+q} y_{n}
$$

with

$$
r=\frac{\alpha}{\beta}, \quad r_{1}=\frac{\alpha_{1}}{\beta_{1}}
$$

and

$$
s=\frac{\beta}{\gamma}, \quad s_{1}=\frac{\beta_{1}}{\gamma_{1}} .
$$

So, in order to study the system (1.7), we investigate the system (1.8). 


\section{Preliminaries}

For the completenessin the paper, we find useful to remind some basic concepts of the difference equations theory as follows:

Let us introduce the six-dimensional discrete dynamical system

$$
\begin{aligned}
& x_{n+1}=f_{1}\left(x_{n}, x_{n-1}, x_{n-2}, y_{n}, y_{n-1}, y_{n-2}\right), \\
& y_{n+1}=f_{2}\left(x_{n}, x_{n-1}, x_{n-2}, y_{n}, y_{n-1}, y_{n-2}\right),
\end{aligned}
$$

$n \in \mathbb{N}$ where $f_{1}: I_{1}^{3} \times I_{2}^{3} \rightarrow I_{1}$ and $f_{2}: I_{1}^{3} \times I_{2}^{3} \rightarrow I_{2}$ are condinuously differentiable functions and $I_{1}, I_{2}$ are some invervals of real numbers. Then, for every initial conditions $\left(x_{i}, y_{i}\right) \in I_{1} \times I_{2}$, for $i=-2,-1,0$ the system (2.1) has a unique solution $\left\{\left(x_{n}, y_{n}\right)\right\}_{n=-2}^{\infty}$.

Definition 2.1. An equilibrium point of stsytem (2.1) is a point $(\bar{x}, \bar{y})$ that satisfies

$$
\begin{gathered}
\bar{x}=f_{1}(\bar{x}, \bar{x}, \bar{x}, \bar{y}, \bar{y}, \bar{y}), \\
\bar{y}=f_{2}(\bar{x}, \bar{x}, \bar{x}, \bar{y}, \bar{y}, \bar{y}),
\end{gathered}
$$

Together with system (2.1), if we consider the associatedvector map

$$
F=\left(f_{1}, x_{n}, x_{n-1}, x_{n-2}, f_{2}, y_{n}, y_{n-1}, y_{n-2}\right),
$$

then the point $(\bar{x}, \bar{y})$ is also called o fixed point of the vector map $F$.

Definition 2.2. If $(\bar{x}, \bar{y})$ be an equilibrium point of a map

$$
F=\left(f_{1}, x_{n}, x_{n-1}, x_{n-2}, f_{2}, y_{n}, y_{n-1}, y_{n-2}\right)
$$

where $f_{1}$ and $f_{2}$ are continuously differentiable functions at $(\bar{x}, \bar{y})$. The linearized system (2.1) about the equilibrium point $(\bar{x}, \bar{y})$

is

$$
X_{n+1}=F\left(X_{n}\right)=B X_{n}
$$

where

$$
X_{n}=\left(\begin{array}{c}
x_{n} \\
x_{n-1} \\
x_{n-2} \\
y_{n} \\
y_{n-1} \\
y_{n-2}
\end{array}\right)
$$

and $B$ is a Jacobian matrix of the system (2.1) about the equilibrium point $(\bar{x}, \bar{y})$.

Theorem 2.3. For the system $X_{n+1}=F\left(X_{n}\right), n=0,1, \ldots$, of difference equations such that $\bar{X}$ is a fixed point of $F$. If all eigenvalues of the Jacobian matrix $B$ about $\bar{X}$ lie inside the open unit disk $|\lambda|<1$, then $\bar{X}$ is locally asymptotically stable. If one of them has a modulus greater then one, then $\bar{X}$ is unstable.

\section{Stability Character Of Equilibrium}

In this section we will prove the stability nature of the zero equilibrium point. In the following theorem we will give the equilibrium points of system (1.8).

Theorem 3.1. For all parameters $r, r_{1}, s, s_{1}$, system (1.8) have a unique zero equilibrium point.

Proof. It is clear from the equilibrium definition. 
Before we give the following stability theorems about the local asymptotic stability of the zero equilibrium point, we build the corresponding linearized form of the system (1.8) and consider the following transformation;

$$
\left(x_{n}, x_{n-1}, x_{n-2}, y_{n}, y_{n-1}, y_{n-2}\right) \rightarrow\left(f, f_{1}, f_{2}, g, g_{1}, g_{2}\right)
$$

where

$$
\begin{aligned}
f & =\frac{r y_{n-1}}{1+s_{1} y_{n}^{p} y_{n-2}^{q}}, \\
f_{1} & =x_{n}, \\
f_{2} & =x_{n-1}, \\
g & =\frac{r_{1} x_{n-1}}{1+s x_{n}^{p} x_{n-2}^{q}}, \\
g_{1} & =y_{n}, \\
g_{2} & =y_{n-1} .
\end{aligned}
$$

The Jacobian matrix about the fixed point $(\bar{x}, \bar{y})$ under the above transformation is as follows:

$$
B(\bar{x}, \bar{y})=\left(\begin{array}{cccccc}
0 & 0 & 0 & -\frac{r s_{1} p \bar{y}^{p+q}}{\left(1+s_{1} \bar{y}^{p+q}\right)^{2}} & \frac{r}{1+s_{1} \bar{y}^{p+q}} & -\frac{r s_{1} q \bar{y}^{p+q}}{\left(1+s_{1} \bar{y}^{p+q}\right)^{2}} \\
1 & 0 & 0 & 0 & 0 & 0 \\
0 & 1 & 0 & 0 & 0 & 0 \\
-\frac{r_{1} s p \bar{x}^{p+q}}{\left(1+s \bar{x}^{p+q}\right)^{2}} & \frac{r_{1}}{1+s \bar{x}^{p+q}} & -\frac{r_{1} s q \bar{x}^{p+q}}{\left(1+s \bar{x}^{p+q}\right)^{2}} & 0 & 0 & 0 \\
0 & 0 & 0 & 1 & 0 & 0 \\
0 & 0 & 0 & 0 & 1 & 0
\end{array}\right)
$$

where $r, s, r_{1}, s_{1}, p, q \in(0, \infty)$.

Theorem 3.2. For system (1.8) the following properties hold:

(i) The zero equilibrium point is locally asymptotically stable if $r r_{1}<1$.

(ii) The zero equilibrium point is locally unstable if $r r_{1}>1$.

Proof. (i) The linearized system of system (1.8) about the equilibrium point

$$
\left(\bar{x}_{0}, \bar{y}_{0}\right)=(0,0)
$$

is given by

$$
X_{n+1}=B\left(\bar{x}_{0}, \bar{y}_{0}\right) X_{n},
$$

where

$$
X_{n}=\left(\begin{array}{c}
x_{n} \\
x_{n-1} \\
x_{n-2} \\
y_{n} \\
y_{n-1} \\
y_{n-2}
\end{array}\right)
$$

and

$$
B\left(\bar{x}_{0}, \bar{y}_{0}\right)=\left(\begin{array}{cccccc}
0 & 0 & 0 & 0 & r & 0 \\
1 & 0 & 0 & 0 & 0 & 0 \\
0 & 1 & 0 & 0 & 0 & 0 \\
0 & r_{1} & 0 & 0 & 0 & 0 \\
0 & 0 & 0 & 1 & 0 & 0 \\
0 & 0 & 0 & 0 & 1 & 0
\end{array}\right) .
$$

The characteristic equation of $B\left(\bar{x}_{0}, \bar{y}_{0}\right)$ is as follows:

$$
P(\lambda)=\lambda^{6}-\left(r r_{1}\right) \lambda^{2}=0 .
$$


The roots of $P(\lambda)$ are

$$
\begin{aligned}
& \lambda_{1,2}=0, \\
& \lambda_{3,4}= \pm \sqrt[4]{r r_{1}}, \\
& \lambda_{5,6}= \pm i \sqrt[4]{r r_{1}} .
\end{aligned}
$$

Since all eigenvalues of the Jacobian matrix $B$ about

$$
\left(\bar{x}_{0}, \bar{y}_{0}\right)=(0,0)
$$

lie inside the open unit disk

$$
|\lambda|<1,
$$

the zero equilibrium point is locally asymptotically stable.

(ii) It is easy to see that if $r r_{1}>1$, then the zero equilibrium point of system (1.8) is unstable.

Now, we will study the global asymptotic stability of system (1.8) about the zero equilibrium point.

Theorem 3.3. The zero equilibrium point of system (1.8) is globally asymptotically stable when $r<1$ and $r_{1}<1$.

Proof. In view of Theorem 3.2, it suffices to prove that

$$
\lim _{n \rightarrow \infty}\left(x_{n}, y_{n}\right)=(0,0) .
$$

It is evident from (1.8) that

$$
0 \leqslant x_{n+1}=\frac{r y_{n-1}}{1+s_{1} y_{n}^{p} y_{n-2}^{q}}<r y_{n-1}<y_{n-1} .
$$

This implies that

$$
x_{4 n+1}<y_{4 n-1}
$$

and

$$
x_{4 n+5}<y_{4 n+3} .
$$

Besides this,

$$
0 \leqslant y_{n+1}=\frac{r_{1} x_{n-1}}{1+s x_{n}^{p} x_{n-2}^{q}}<r_{1} x_{n-1}<x_{n-1}
$$

This implies that

$$
y_{4 n+1}<x_{4 n-1}
$$

and

$$
y_{4 n+5}<x_{4 n+3} .
$$

So

$$
x_{4 n+5}<y_{4 n+3}<x_{4 n+2}
$$

and

$$
y_{4 n+5}<x_{4 n+3}<y_{4 n+2} .
$$

Hence, the subsequences

$$
\left\{x_{4 n+1}\right\},\left\{x_{4 n+2}\right\},\left\{x_{4 n+3}\right\},\left\{x_{4 n+4}\right\}
$$


and

$$
\left\{y_{4 n+1}\right\},\left\{y_{4 n+2}\right\},\left\{y_{4 n+3}\right\},\left\{y_{4 n+4}\right\}
$$

are decreasing. Therefore the sequences $\left\{x_{n}\right\}$ and $\left\{y_{n}\right\}$ are monotonic which are decreasing. Hence

$$
\lim _{n \rightarrow \infty} x_{n}=0
$$

and

$$
\lim _{n \rightarrow \infty} y_{n}=0 .
$$

This completes the proof.

\section{Prime Periodic Two-Solutions 1.8}

In this section we will investigate the periodic nature of system (1.8).

Theorem 4.1. System (1.8) has no prime period two solutions.

Proof. Assuming

$$
\ldots,(a, b),(c, d),(a, b),(c, d), \ldots
$$

is prime period two solutions of the system (1.8) such that

$$
a, b, c, d \neq 0
$$

and

$$
a \neq c, b \neq d .
$$

Then we have

$$
a=\frac{r b}{1+s_{1} d^{p+q}}, b=\frac{r_{1} a}{1+s c^{p+q}}
$$

and

$$
c=\frac{r d}{1+s_{1} b^{p+q}}, d=\frac{r_{1} c}{1+s a^{p+q}}
$$

After some tedious calculations from (4.1) and (4.2), we can obtain the following equilities;

$$
(a+c)^{2}-4 a c=0,
$$

and

$$
(b+d)^{2}-4 b d=0 .
$$

But they are contrary to our assumption and therefore system (1.8) has no prime period-two solutions. This completes the proof.

\section{Rate of Convergence}

In this section, we will give exact results about the rate of convergence of positive solutions that converge to the equilibrium point of the system (1.8), in the regions of parameters described in Theorem (3.3).

Consider the following system of difference equations

$$
\left.\begin{array}{l}
x_{n+1}=f_{1}\left(x_{n}, y_{n}\right), n=0,1, \ldots \\
y_{n+1}=f_{2}\left(x_{n}, y_{n}\right), n=0,1, \ldots
\end{array}\right\}
$$


where $f_{1}, f_{2}$ are continuous functions that maps some set $I$ into $I$. The set $I$ is an interval of real numbers. System (5.1) is competitive if $f_{1}(x, y)$ is non-decreasing in $x$ and non-increasing in $y$ and $f_{2}(x, y)$ is non-increasing in $x$ and non-decreasing in $y$. System (5.1) is called anti-competitive system, if the functions $f_{1}$ and $f_{2}$ have monotonic character opposite to the monotonic character in competitive system.

We state that the following theorems give precise information about the asymptotics of linear non-autonomous difference equations. Consider the scalar $m t h$-order linear difference equation

$$
y_{n+m}+p_{1}(n) y_{n+m-1}+p_{m}(n) y_{n}=0
$$

where $m$ is a positive integer and $p_{i}: \mathbb{Z}^{+} \rightarrow \mathbb{C}$ for $i \in\{1, \ldots, m\}$. Suppose that

$$
q_{i}=\lim _{n \rightarrow \infty} p_{i}(n), \text { for } i=1,2, \ldots, m,
$$

exist in $\mathbb{C}$. For the following limitting equation of $(5.2)$

$$
y_{n+m}+q_{1} y_{n+m-1}+\ldots+q_{m} y_{n}=0,
$$

the asymptotics of solutions of (5.2) are given the following results. See [25].

Theorem 5.1. (Poincaré's Theorem) Consider (5.2) based on the condition (5.3). Let $\lambda_{i}$ for $i=1, \ldots, m$ be the roots of the characteristic equation

$$
\lambda^{m}+q_{1} \lambda^{m-1}+\ldots+q_{m}=0
$$

of the limiting equation (5.4) under the condition that $\left|\lambda_{i}\right| \neq\left|\lambda_{j}\right|$ for $i \neq j$. If $x_{n}$ is a positive solution of (5.2), then either $x_{n}=0$ for all large $n$ or there exists an index $j \in\{1, \ldots, m\}$ such that

$$
\lim _{n \rightarrow \infty} \frac{x_{n+1}}{x_{n}}=\lambda_{j}
$$

The releated results were obtained by Perron, and one of Perron's results was improved by Pituk, see [25].

Theorem 5.2. Assume that (5.3) holds. If $x_{n}$ is a positive solution of (5.2), then either eventually $x_{n}=0$ or

$$
\lim _{n \rightarrow \infty} \sup \left(\left|x_{n j}\right|\right)^{1 / n}=\left|\lambda_{j}\right|,
$$

where $\lambda_{1}, \ldots, \lambda_{m}$ are the roots (not necessarily distinct) of the characteristic equation (5.5).

Consider

$$
Y_{n+1}=[A+B(n)] Y_{n}
$$

where $Y_{n}$ is an $m$-dimensional vector, $A \in C^{m \times m}$ is a constant matrix and

$$
B: \mathbb{Z}^{+} \rightarrow C^{m \times m}
$$

is a matrix function satisfying

$$
\|B(n)\| \rightarrow 0 \text {, when } n \rightarrow \infty,
$$

where $\|$.$\| denotes any matrix norm which is associated with the vector norm \|$.$\| . See [20].$

Theorem 5.3. (Pituk) Suppose that condition (5.7) holds for system (5.6). If $Y_{n}$ is a solution of (5.6), then either

$$
Y_{n}=0
$$

for all large $n$ or

$$
\theta=\lim _{n \rightarrow \infty}\left\|Y_{n}\right\|^{1 / n}
$$

exists and $\theta$ is equal to the modulus of one the eigenvalues of the matrix A. 
Theorem 5.4. (Pituk) Suppose that condition (5.7) holds for system (5.6). If $Y_{n}$ is a solution of (5.6), then either

$$
Y_{n}=0
$$

for all large $n$ or

$$
\theta=\lim _{n \rightarrow \infty} \frac{\left\|Y_{n+1}\right\|}{\left\|Y_{n}\right\|}
$$

exists and $\theta$ is equal to the modulus of one the eigenvalues of the matrix $A$.

Using Theorem (5.3) and (5.4), we obtain the following rate of convergence result.

Theorem 5.5. Suppose that $r<1$ and $r_{1}<1$. Let $\left\{\left(x_{n}, y_{n}\right)\right\}_{n=-2}^{\infty}$ be any positive solution of the system (1.8) such that

$$
\begin{aligned}
& \lim _{n \rightarrow \infty} x_{n}=\bar{x}_{1}, \\
& \lim _{n \rightarrow \infty} y_{n}=\bar{x}_{2}
\end{aligned}
$$

where $M=\left(\bar{x}_{1}, \bar{x}_{2}\right)$ and $M$ is globally asymptotically stable. Then, the error vector

$$
E_{n}=\left(\begin{array}{c}
e_{n}^{1} \\
e_{n-1}^{1} \\
e_{n-2}^{1} \\
e_{n}^{2} \\
e_{n-1}^{2} \\
e_{n-2}^{2}
\end{array}\right)_{6 \times 1}=\left(\begin{array}{c}
x_{n}-\bar{x}_{1} \\
x_{n-1}-\bar{x}_{1} \\
x_{n-2}-\bar{x}_{1} \\
y_{n}-\bar{x}_{2} \\
y_{n-1}-\bar{x}_{2} \\
y_{n-2}-\bar{x}_{2}
\end{array}\right)_{6 \times 1}
$$

of every positive solution of the system (1.8) satisfies both of the following asymptotic relations:

$$
\begin{aligned}
& \lim _{n \rightarrow \infty}\left\|E_{n}\right\|^{1 / n}=\left|\lambda_{i} J_{F}(M)\right|, \text { for some } i=1,2, \ldots, 6 \\
& \lim _{n \rightarrow \infty} \frac{\left\|E_{n+1}\right\|}{\left\|E_{n}\right\|}=\left|\lambda_{i} J_{F}(M)\right|, \text { for some } i=1,2, \ldots, 6
\end{aligned}
$$

where

$$
\left|\lambda_{i} J_{F}(M)\right|
$$

is equal to the modulus of one the eigenvalues of the Jacobian matrix evaluated at the equilibrium point M.

Proof. Let $\left\{\left(x_{n}, y_{n}\right)\right\}_{n=-2}^{\infty}$ be any positive solution of the system (1.8) such that

$$
\lim _{n \rightarrow \infty} x_{n}=\bar{x}_{1}
$$

and

$$
\lim _{n \rightarrow \infty} y_{n}=\bar{x}_{2} .
$$

To find the error terms, we have

$$
\begin{aligned}
& x_{n+1}-\bar{x}_{1}=\sum_{i=0}^{2} A_{i}\left(x_{n-i}-\bar{x}_{1}\right)+\sum_{i=0}^{2} B_{i}\left(y_{n-i}-\bar{x}_{2}\right) \\
& y_{n+1}-\bar{x}_{2}=\sum_{i=0}^{2} C_{i}\left(x_{n-i}-\bar{x}_{1}\right)+\sum_{i=0}^{2} D_{i}\left(y_{n-i}-\bar{x}_{2}\right) .
\end{aligned}
$$

Set

$$
\begin{aligned}
& e_{n}^{1}=x_{n}-\bar{x}_{1}, \\
& e_{n}^{2}=y_{n}-\bar{x}_{2} ;
\end{aligned}
$$

therefore, it follows that

$$
\begin{aligned}
& e_{n+1}^{1}=\sum_{i=0}^{2} A_{i} e_{n-i}^{1}+\sum_{i=0}^{2} B_{i} e_{n-i}^{2} \\
& e_{n+1}^{2}=\sum_{i=0}^{2} C_{i} e_{n-i}^{1}+\sum_{i=0}^{2} D_{i} e_{n-i}^{2}
\end{aligned}
$$


where

$$
\begin{aligned}
& A_{0}=0, A_{1}=0, \quad A_{2}=0, \\
& B_{0}=-\frac{r s_{1} p \bar{y}\left(y_{n}^{p} y_{n-2}{ }^{q}-\bar{y}^{p+q}\right)}{\left(1+s_{1} y_{n}^{p} y_{n-2}\right)^{2}}, \\
& B_{1}=\frac{r}{1+s_{1} y_{n}^{p} y_{n-2} q}, \\
& B_{2}=-\frac{r s_{1} q \bar{y}\left(y_{n}^{p} y_{n-2}{ }^{q}-\bar{y}^{p+q}\right)}{\left(1+s_{1} y_{n}^{p} y_{n-2}\right)^{2}}, \\
& C_{0}=-\frac{r_{1} s p \bar{x}\left(x_{n}^{p} x_{n-2}{ }^{q}-\bar{x}^{p+q}\right)}{\left(1+s x_{n}^{p} x_{n-2}\right)^{2}}, \\
& C_{1}=\frac{r_{1}}{1+s x_{n}^{p} x_{n-2} q}, \\
& C_{2}=-\frac{r_{1} s q \bar{x}\left(x_{n}^{p} x_{n-2}{ }^{q}-\bar{x}^{p+q}\right)}{\left(1+s x_{n}^{p} x_{n-2}\right)^{2}}, \\
& D_{0}=0, \quad D_{1}=0, \quad D_{2}=0 .
\end{aligned}
$$

Taking the limits, it is clear that

$$
\begin{aligned}
& \lim _{n \rightarrow \infty} A_{0}=0, \lim _{n \rightarrow \infty} A_{1}=0, \lim _{n \rightarrow \infty} A_{2}=0, \\
& \lim _{n \rightarrow \infty} B_{0}=-\frac{r s_{1} p \bar{y}\left(y_{n}^{p} y_{n-2}{ }^{q}-\bar{y}^{p+q}\right)}{\left(1+s_{1} y_{n}^{p} y_{n-2}\right)^{2}}, \\
& \lim _{n \rightarrow \infty} B_{1}=\frac{r}{1+s_{1} y_{n}^{p} y_{n-2} q}, \\
& \lim _{n \rightarrow \infty} B_{2}=-\frac{r s_{1} q \bar{y}\left(y_{n}^{p} y_{n-2}{ }^{q}-\bar{y}^{p+q}\right)}{\left(1+s_{1} y_{n}^{p} y_{n-2}\right)^{2}}, \\
& \lim _{n \rightarrow \infty} C_{0}=-\frac{r_{1} s p \bar{x}\left(x_{n}^{p} x_{n-2}{ }^{q}-\bar{x}^{p+q}\right)}{\left(1+s x_{n}^{p} x_{n-2}\right)^{2}}, \\
& \lim _{n \rightarrow \infty} C_{1}=\frac{r_{1}}{1+s x_{n}^{p} x_{n-2} q}, \\
& \lim _{n \rightarrow \infty} C_{2}=-\frac{r_{1} s q \bar{x}\left(x_{n}^{p} x_{n-2} q-\bar{x}^{p+q}\right)}{\left(1+s x_{n}^{p} x_{n-2}\right)^{2}}, \\
& \lim _{n \rightarrow \infty} D_{0}=0, \lim _{n \rightarrow \infty} D_{1}=0, \lim _{n \rightarrow \infty} D_{2}=0 .
\end{aligned}
$$

That is

$$
\begin{aligned}
& B_{0}=-\frac{r s_{1} p \bar{y}\left(y_{1}^{p} y_{2}^{q}-\bar{y}^{p+q}\right)}{\left(1+s_{1} \bar{y}_{2}^{p+q}\right)^{2}}+\alpha_{n}, \\
& B_{1}=\frac{r}{1+s_{1} \bar{y}_{2}^{p+q}+\beta_{n},} \\
& B_{2}=-\frac{r s_{1} q \bar{y}\left(y_{1}^{p} y_{2}^{q}-\bar{y}^{p+q}\right)}{\left(1+s_{1} \bar{y}_{2}^{p+q}\right)^{2}}+\gamma_{n}, \\
& C_{0}=-\frac{r_{1} s p \bar{x}\left(x_{2}^{p} x_{1}^{q}-\bar{x}^{p+q}\right)}{\left(1+s \bar{x}_{1}^{p+q}\right)^{2}}+\delta_{n}, \\
& C_{1}=\frac{r_{1}}{1+s \bar{x}_{1}^{p+q}+\eta_{n},} \\
& C_{2}=--\frac{r_{1} s q \bar{x}\left(x_{2}^{p} x_{1}^{q}-\bar{x}^{p+q}\right)}{\left(1+s \bar{x}_{1}^{p+q}\right)^{2}}+\theta_{n},
\end{aligned}
$$


where $\alpha_{n} \rightarrow 0, \beta_{n} \rightarrow 0, \gamma_{n} \rightarrow 0, \delta_{n} \rightarrow 0, \eta_{n} \rightarrow 0, \theta_{n} \rightarrow 0$ for $n \rightarrow \infty$.

Thus, the limitting system of error terms about the equilibrium $M$ can be written as follows:

$$
E_{n+1}=(C+D(n)) E_{n}
$$

where $E_{n}=\left(e_{n}^{1}, e_{n-1}^{1}, e_{n-2}^{1}, e_{n}^{2}, e_{n-1}^{2}, e_{n-2}^{2}\right)^{T}$,

$$
\begin{aligned}
C & =\left(\begin{array}{cccccc}
0 & 0 & 0 & 0 & r & 0 \\
1 & 0 & 0 & 0 & 0 & 0 \\
0 & 1 & 0 & 0 & 0 & 0 \\
0 & r_{1} & 0 & 0 & 0 & 0 \\
0 & 0 & 0 & 1 & 0 & 0 \\
0 & 0 & 0 & 0 & 1 & 0
\end{array}\right)_{6 \times 6}, \\
D_{n} & =\left(\begin{array}{cccccc}
0 & 0 & 0 & \alpha_{n} & \beta_{n} & \gamma_{n} \\
0 & 0 & 0 & 0 & 0 & 0 \\
0 & 0 & 0 & 0 & 0 & 0 \\
\delta_{n} & \eta_{n} & \theta_{n} & 0 & 0 & 0 \\
0 & 0 & 0 & 0 & 0 & 0 \\
0 & 0 & 0 & 0 & 0 & 0
\end{array}\right)_{6 \times 6}
\end{aligned}
$$

and $\|D(n)\| \rightarrow 0$, when $n \rightarrow \infty$. As desired.

Corollary 5.6. Assume that $r r_{1}<1$. Then, the error vector of every non-trivial solution of system 1.8 satisfies both of the following asymptotic relations:

$$
\begin{aligned}
& \lim _{n \rightarrow \infty}\left\|E_{n}\right\|^{1 / n}=\left|\lambda_{i} J_{F}(M)\right|, \text { for some } i=1,2,3,4,5,6, \\
& \lim _{n \rightarrow \infty} \frac{\left\|E_{n+1}\right\|}{\left\|E_{n}\right\|}=\left|\lambda_{i} J_{F}(M)\right|, \text { for some } i=1,2,3,4,5,6
\end{aligned}
$$

where $\left|\lambda_{i} J_{F}(M)\right|$ is equal to the modulus of one the eigenvalues of the Jacobian matrix evaluated at the equilibrium point M, i.e. $\left\{\lambda_{1,2}=0, \lambda_{3,4}= \pm \sqrt[4]{r r_{1}}, \lambda_{5,6}= \pm i \sqrt[4]{r r_{1}}\right.$.

\section{Conclusions}

In the present paper, we described the qualitative behaviors of solutions of the system (1.8) of nonlinear difference equations. More precisely, we studied the equilibrium points, the local asymptotic stability, the global asymptotic stability of zero equilibrium, the existence of the prime two-periodic solutions and the rate of convergence of positive solutions of the aforementioned system. Also, we gave a correction about an article in the literature. Our system generalized the systems studied in $[13,14,27]$.

The results in this paper can be extend to the following system of difference equations;

$$
\left.\begin{array}{c}
u_{n+1}=\frac{\alpha v_{n-1}}{\beta+\gamma \sum_{i=0}^{k} v_{n-2 i}^{p_{i}}}, \\
v_{n+1}=\frac{\alpha_{1} u_{n-1}}{\beta_{1}+\gamma_{1} \sum_{i=0}^{k} u_{n-2 i}^{p_{i}}}
\end{array}\right\}, n=0,1, \ldots
$$

\section{Conflict of Interests}

The author declares that there is no conflict of interests regarding the publication of this manuscript. 


\section{References}

[1] R. Agarwal, Difference equations and inequalities, theory, methods and applications, Marcel Dekker Inc., New York 1992.

[2] R. Abo-Zeid and H. Kamal, Global behavior of two rational third order difference equations, Univers. J. Math. Appl., 2(4) (2019), 212-217.

[3] S. Abualrub and M. Aloqeili, Dynamics of the System of Difference Equations $x_{n+1}=A+y_{n-k} / y_{n}, y_{n+1}=B+x_{n-k} / x_{n}$, Qual. Theory Dyn. Syst., 19(2) (2020), 1-19.

[4] A. M. Ahmed, On the Dynamics of Higher-Order Rational Difference Equation, Discrete Dyn. Nat. Soc., 2011, Article ID: 419789, 8 pages.

[5] A. M. Ahmed, The Dynamics of the Recursive Sequences $x_{n+1}=\frac{b x_{n-1}}{A+B x_{n}^{p} x_{n-2}^{p}}$, J.Pure Appl. Math.: Adv. Applic., 1(2) (2009), 215-223.

[6] M. M. Alzubaidi and E. M. Elsayed, Analytical and Solutions of Fourth Order Difference Equations, Commun. Adv. Math. Sci., 2(1) (2019), 9-21.

[7] Q. Din, T. F. Ibrahim and A.Q. Khan, Behavior of a competitive system of second order difference equations, Sci. World J., Article ID: 283982.

[8] Q. Din, Asymptotic behavior of an anti-competitive system of second-order difference equations, J. Egyptian Math. Soc., 24(1) (2016), 37-43.

[9] H. M. El-Owaidy, A. M. Ahmed and A. M. Youssef, The Dynamics of the Recursive Sequence $x_{n+1}=\frac{\alpha x_{n-1}}{\beta+\gamma x_{n-2}^{p}}$, Appl. Math. Lett., 18 (2005), 1013-1018.

[10] M. M. El-Dessoky, E. M. Elsayed, E. M. Elabbasy and A. Asiri, Expressions of the solutions of some systems of difference equations, J. Comput. Anal. Appl., 27(7) (2019), 1161-1172.

[11] S. Elaydi, An Introduction to Difference Equations, 3rd ed., Springer-Verlag, New York, 2005.

[12] M. Gocen and M. Guneysu, The Global Attractivity of some ratinal difference equations, J. Comp. Anal. Appl., 25 (7) (2018), 1233-1243.

[13] M. Gümüş and Y. Soykan, Global Character of a Six-Dimensional Nonlinear System of Difference Equations, Discrete Dyn. Nat. Soc., 2016, Article ID 6842521.

[14] M. Gümüş and Ö. Öcalan, The Qualitative Analysis of a Rational System of Difference Equations, J. Fract. Calc. Appl., 9(2) (2018), 113-126.

[15] M. Gümüş and Y. Soykan, Dynamics of Positive Solutions of a Higher Order Fractional Difference Equation with Arbitrary Powers, J. Appl. Math. Inf., 35(3-4) (2017), 267-276.

[16] V. Kocic and G. Ladas, Global behavior of nonlinear difference equations of higher order with applications, Kluwer Academic Publishers, Dordrecht, (1993).

[17] F. H. Gümüş, Yüksek Mertebeden Fark Denklemlerinin Global Davranışları Üzerine, Master Thesis, Afyon Kocatepe Üniversitesi Fen Bilimleri Enstitüsü Afyon (2015).

[18] A. Khelifa, Y. Halim and M. Berkal, Solutions of a system of two higher-order difference equations in terms of lucas sequence, Univers. J. Math. Appl., 2(4) (2019), 202-211.

[19] M. A. Kerker, E. Hadidi, and A. Salmi, Qualitative behavior of a higher-order nonautonomous rational difference equation, J. Appl. Math. Comput., 64 (2020), 399-409.

[20] M. R. S. Kulenovic and O. Merino, Discrete Dynamical Systems and Difference Equations with Mathematica. Chapman and Hall/CRC, Boca Raton, London. (2002).

[21] M. R. S. Kulenović and G. Ladas, Dynamics of second order rational difference equations, Chapman and Hall/CRC, (2001).

[22] M. Kara, N. Touafek and Y. Yazlik, Well-Defined Solutions of a Three-Dimensional System of Difference Equations, Gazi Univ. J.Sci., 33(3), 767-778.

[23] W. X. Ma, Global Behavior of an Arbitrary-Order Nonlinear Difference Equation with a Nonnegative Function, Mathematics, 8(5) (2020), 825.

[24] M. Merdan and Ş. Şişman, Investigation of linear difference equations with random effects, Adv. Difference Equ., 2020 (1) (2020), 1-19. 
[25] M. Pituk, More on Poincares and Perrons Theorems for Difference Equations, J. Difference Equ. Applic., 8(3) (2002), 201-216.

[26] A. Sanbo, E. M. Elsayed and F. Alzahrani, Dynamics of the nonlinear rational difference equation $x_{n+1}=\left(A x_{n-\alpha} x_{n-\beta}+\right.$ $\left.B x_{n-\gamma}\right) /\left(C x_{n-\alpha} x_{n-\beta}+D x_{n-\gamma}\right)$, Indian J. Pure Appl. Math., 50(2) (2019), 385-401.

[27] M. N. Qureshi and A. Q. Khan, Global dynamics of $(1,2)$ - type systems of difference equations, Malaya J. Matematik, 6(2), 408-416.

[28] M. N. Qureshi, Q. Din and A. Q. Khan, Asymptotic behavior of an anti-competitive system of rational difference equations, Life Sci. J., 11(2014): 1-7.

[29] M. N. Qureshi and Q. Din, Oualitative behavior of an anti-competitive system of third-order rational difference equations, Comput. Ecology Software, 4(2) (2014), 104-115.

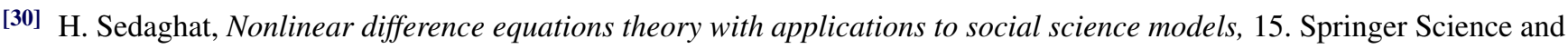
Business Media, (2003).

[31] D. T. Tollu and I. Yalçınkaya, Global behavior of a three dimensional system of difference equations of order three, Commun. Fac. Sci. Univ. Ank. Ser. A1 Math. Stat., 68(1) (2019), 1-16.

[32] I. Yalçınkaya, On the Global Asymptotic Stability of a Second-Order System of Difference Equations, Discrete Dyn. Nat. Soc., 2008, Article ID 860152.

[33] D. Zhang, W. Ji, L. Wang and X. Li, On the symmetrical system of rational difference equation $x_{n+1}=A+y_{n-k} / y_{n}$, $y_{n+1}=A+x_{n-k} / x_{n}$, Appl. Math., 4 (2013), 834-837. 